\title{
Hemorragia de la matriz germinal: puede ser detectada de manera efectiva
}

\section{Germinal matrix hemorrhage: can be detected effectively}

\author{
Juan Carlos Jara Santamaría ${ }^{1}$, Luis Ernesto Torres Morán ${ }^{2}$, Cecilia Carmen Trujillo \\ Jácome $^{3}$, Angel Patricio Yánez Moretta ${ }^{4}$, Guido Fernando Cumbicus Naranjo ${ }^{5}$ \& \\ Shirley Gabriela Mantilla Naranjo ${ }^{6}$
}

\section{DOI: https://doi.org/10.33262/anatomiadigital.v3i3.1328}

\begin{abstract}
The germinal matrix hemorrhage corresponds to a cerebrovascular event originated in its blood vessels, it is prevalent in premature babies and is the first cause of infant mortality in Ecuador; Its inadequate diagnosis has an important impact on the neurodevelopment of premature infants and can generate serious sequelae that may be greater depending on the degree of prematurity, especially in premature infants weighing 500 to $750 \mathrm{~g}$ at birth. One of the main tools to diagnose womb bleeding is
\end{abstract}

Resumen La hemorragia de la matriz germinal corresponde a un evento cerebrovascular originado en sus vasos sanguíneos, es prevalente en los prematuros y es la primera causa de mortalidad infantil en Ecuador; su inadecuado diagnóstico tiene un importante impacto en el neurodesarrollo de los prematuros y puede generar secuelas graves que pueden ser mayores conforme al grado de prematuridad, especialmente en prematuros con un peso de 500 a $750 \mathrm{~g}$ al nacimiento. Una de las principales herramientas para

\footnotetext{
${ }^{1}$ Hospital del IESS Quito Sur, Médico Tratante. Universidad de las Américas: Facultad de Ciencias de la Salud, Quito, Ecuador. iD https://orcid.org/0000-0001-9708-5407 Juantroy@ hotmail.es

${ }^{2}$ Universidad San Francisco de Quito, Profesor del Posgrado de Radiología. Quito, Ecuador.

(iD https://orcid.org/0000-0002-1927-3207 luis550504@gmail.com

${ }^{3}$ Hospital de Especialidades "Eugenio Espejo”, Médico Tratante. Quito, Ecuador.

(iD) ceciliatrujillojacome@yahoo.es

${ }^{4}$ Universidad Internacional del Ecuador, Escuelas de Biología Aplicada y de Gestión Ambiental, Quito, Ecuador. iD https://orcid.org/0000-0003-4436-7632 apyanez@hotmail.com; ayanez@uide.edu.ec

${ }^{5}$ Universidad de las Américas, Facultad de Ciencias de la Salud, Quito, Ecuador.

(iD https://orcid.org/0000-0003-0988-8563 Fernadhino001@hotmai.com

${ }^{6}$ Universidad de las Américas, Facultad de Ciencias de la Salud, Quito, Ecuador.

(iD) https://orcid.org/0000-0002-5710-1537 gabi12022009@hotmail.com
} 
transfontanelar ultrasound, which is fundamental in the detection and early management of the situation. It should be considered that it requires an experienced radiologist to identify and interpret the findings. Some associated risk factors are: gestational age, low birth weight, low Apgar score, acidosis, and neonatal asphyxia. The factors that are involved in bleeding can be: intravascular, vascular and extravascular. The degrees can range from Grade I (hyperechogenic mass due to the presence of clots, the choroid plexus looks thickened in the trigone region), Grade II (in which the hemorrhage extends to the cisterna magna, which increases the risk of hydrocephalus), Grade III in which the clot will become more anechoic over time, the presence of blood in the CSF can cause chemical ventriculitis) and Grade IV (with paraventricular hypercogenecity with involvement of the frontal and parietal lobes). Transfontanelar ultrasound can detect various brain lesions in the premature newborn, including bleeding from the germ matrix and periventricular leukomalacia. The main cause of leukomalacia is hypoxicischemic events, so that the lesions predominate in the periventricular white matter. The use of this technique is recommended for the timely diagnosis of bleeding in newborns with risk factors.

Keywords: Hemorrhage, germinal matrix, infant mortality, transfontanelar ultrasound, Ecuador.

\section{Introducción}

La hemorragia de la matriz germinal (HMG) es una entidad prevalente en los prematuros. El riesgo de nacimientos prematuros para la población general en el mundo se estima diagnosticar la hemorragia de la matriz es el ultrasonido transfontanelar que es fundamental en la detección y manejo temprano la situación, se debe considerar que requiere de un radiólogo experimentado para identificar e interpretar los hallazgos. Los factores de riesgo asociados son: la edad gestacional, el peso bajo al nacer, la puntuación baja del test de Apgar, la acidosis y la asfixia neonatal. Los factores que están implicados en la hemorragia pueden ser: intravasculares, vasculares y extravasculares. Los grados pueden ir del Grado I (masa hiperecogénica por la presencia de coágulos, el plexo coroideo luce engrosado en la región del trígono), Grado II (en el que la hemorragia se extiende hacia la cisterna magna lo que incrementa el riesgo de hidrocefalia), Grado III en el que el coágulo se volverá más anecoico con el tiempo, la presencia de sangre en el LCR puede producir una ventriculitis química) y Grado IV (con hipercogenecidad paraventricular con afectación de lóbulos frontales y parietales). El ultrasonido transfontanelar puede detectar varias lesiones cerebrales en el recién nacido prematuro entre estas la hemorragia de la matriz germinal y la leucomalacia periventricular. La causa principal de la leucomalacia son los eventos hipóxicos-isquémicos, por lo que las lesiones predominan en la sustancia blanca periventricular. Se recomienda el uso de esta técnica para el diagnóstico oportuno de la hemorragia en los recién nacidos que presenten factores de riesgo.

Palabras claves: Hemorragia, matriz germinal, mortalidad infantil, ultrasonido transfontanelar, Ecuador. 
entre el $6 \%$ y $10 \%$. Es la primera causa de mortalidad infantil en el país, según datos del INEC, por lo que es un tema de vital importancia en Ecuador, dado su impacto en el neurodesarrollo de los prematuros y las secuelas graves que desencadena su inadecuado diagnóstico y manejo, produciendo así una afectación en la economía familiar y a su vez en la salud pública (MSP, 2015).

La matriz subependimaria, matriz germinal o eminencia gangliónica se localiza entre el núcleo caudado y el tálamo a nivel del foramen de Monro, está representada por una red capilar localizada en la zona limítrofe entre las arterias y las venas profundas del encéfalo, demuestra alta vulnerabilidad a las variaciones de presión e inestabilidad hemodinámica (Ballabh, 2010).

La hemorragia de la matriz germinal es un evento cerebro vascular cuyo principal origen está en variaciones de sus vasos sanguíneos, altera el revestimiento ependimario y podría extenderse a nivel intraventricular, particularmente a los laterales. Es la patología de mayor frecuencia entre los trastornos hemorrágicos intracraneales neonatales (Segovia et $a l ., 2003$ ), incrementa conforme el grado de prematuridad en prematuros con un peso de 500 a $750 \mathrm{~g}$ y puede aumentar su incidencia en tales casos hasta en un 45\% (Ballabh, 2010; Cervantes-Ruiz et al., 2012).

Una de las principales herramientas para diagnosticar la hemorragia de la matriz germinal es el ultrasonido transfontanelar, es una herramienta operador-dependiente, requiere de un radiólogo experimentado para poder identificar e interpretar los hallazgos ecográficos adecuadamente.

Por lo que resulta imperioso que los médicos generales conozcan del tema, los especialistas en imagen lo diagnostiquen eficientemente y los pediatras neonatólogos realicen un manejo adecuado a partir de un análisis clínico radiológico oportuno, igual que en circunstancias similares en las que el conocimieno y manejo adecuado de la imagenología son fundamentales (Jara-Santamaría et al., 2018; Montalvo-Ramos et al., 2018).

\section{El desarrollo embriológico del sistema nervioso central}

Es necesario recordar la evolución morfológica del Sistema Nervioso Central (SNC), reconocer los cambios que se producen durante las etapas de su formación y comprender los procesos patológicos que pueden ocurrir en estas etapas.

El SNC se forma a partir del ectodermo embrionario, en la tercera semana pasa por las etapas de placa, surco y tubo neural, tiene dos porciones bien definidas una anterior dilatada en forma de tres vesículas que formarán el encéfalo y otra posterior tubular que formará la médula espinal. En la quinta semana, el embrión tiene cinco vesículas encefálicas: el telencéfalo y el diencéfalo derivados del prosencéfalo (cerebro anterior), el mesencéfalo (medio) que permanece indiviso y el metencéfalo y mielencéfalo que derivaron del rombencéfalo (cerebro posterior) (Moore, 2009). 
La embriología de la corteza cerebral es un proceso complejo, todavía actualmente no bien conocido, que puede dividirse en tres etapas fundamentales: proliferación celular, migración celular y organización cortical.

Proliferación celular: en torno a la séptima semana del desarrollo embrionario se produce una proliferación de neuronas jóvenes en la capa subependimaria de las paredes de los ventrículos laterales. Esta área de proliferación celular se denomina matriz germinal, y en ella tiene lugar la mitosis de las células madre para la producción de las neuronas y células gliales que formarán el cerebro maduro. Algunas de estas nuevas células formadas en la mitosis permanecerán en la matriz germinal dando lugar a nuevas mitosis, mientras que otras migrarán hacia su destino cortical (Montejo, et al., 2014).

Migración neuronal: en la octava semana gestacional comienza la migración de las primeras neuronas jóvenes desde la matriz germinal para formar el córtex cerebral. Para ello, ocurre elongación de las células de la matriz germinal, colocándose el núcleo en el extremo más alejado de la superficie ventricular. Posteriormente, la mayoría de las neuronas que formarán el córtex migrarán utilizando como guía las células gliales radiales que se agrupan en fascículos de 4 a 10 células y atraviesan el espesor del hemisferio desde la superficie ventricular hasta la piamadre. Estos fascículos de células gliales proporcionarán los metabolitos necesarios para la migración (Montejo et al, 2014).

Organización cortical: La migración sigue un orden establecido que condiciona la posterior organización celular (Montejo et al., 2014).

El SNC difiere del resto de sistemas porque el desarrollo anatómico y funcional se mantiene durante toda la gestación y prosigue después del nacimiento. Por esta razón está expuesto a que se produzcan alteraciones importantes y malformaciones por el efecto de lesiones o por la presentación de situaciones de riesgo de diferente naturaleza (infecciones, traumatismos, hipoxia, etc.) en cualquier edad gestacional. La detección prenatal está sujeta a los siguientes determinantes:

- Los patrones de normalidad cambian con la edad gestacional.

- Las lesiones son progresivas. Pasa un tiempo desde que se ha presentado la situación de riesgo hasta que la anomalía se evidencia en la imagen.

- Una ecografía normal en las semanas 20-22 no excluye a una patología que se pueda presentar posteriormente.

\section{Patogenia y factores de riesgo de la hemorragia de la matriz germinal}

Los principales factores de riesgo son la edad gestacional, el bajo peso al nacer, se han descrito como relevantes otros factores como la baja puntuación del test de Apgar, la acidosis y la asfixia neonatal (Ertan et al., 2006). 
Los factores implicados en la patogenia de la hemorragia de la matriz germinal subependimaria se suelen describir en 3 grupos:

Factores intravasculares: corresponden a las variaciones del volumen y del flujo vascular cerebral, a su vez favorecidas por la alteración de la autorregulación cerebrovascular propia del cerebro del niño prematuro. Estas variaciones ocurren en situaciones como: la hipercarbia, la alta concentración de oxígeno inspirado, la ventilación mecánica, las expansiones de volemia, la hipotensión, el empleo de drogas vasoactivas, la anemia, la hipoglucemia y la hipoxia-isquemia (Cabañas \& Pellicer, 2008).

El aumento de la presión venosa cerebral que sucede en el trabajo de parto y en la rotación lateral de la cabeza también supone mayor riesgo de hemorragia.

Factores vasculares: La anatomía de los capilares de la matriz germinal, fragilidad capilar, alto requerimiento metabólico y su localización en una zona vascular limítrofe entre las arterias talámica y estriadas. Adicionalmente, los vasos se encuentran afectados en la hipoxia-isquemia, la corioamnionitis y la sepsis. En varios estudios se sugiere que la hemorragia tiene su origen en el sistema venoso, sin descartar un posible origen arterial (Figura 1).

Figura 1. El paciente pretérmino posee vascularidad centrípeta, la zona más vulnerable es periventricular, es limítrofe entre las arterias talámicas y estriadas. El paciente a término revela vascularidad centrífuga, la región vulnerable es parasagital y subcortical.

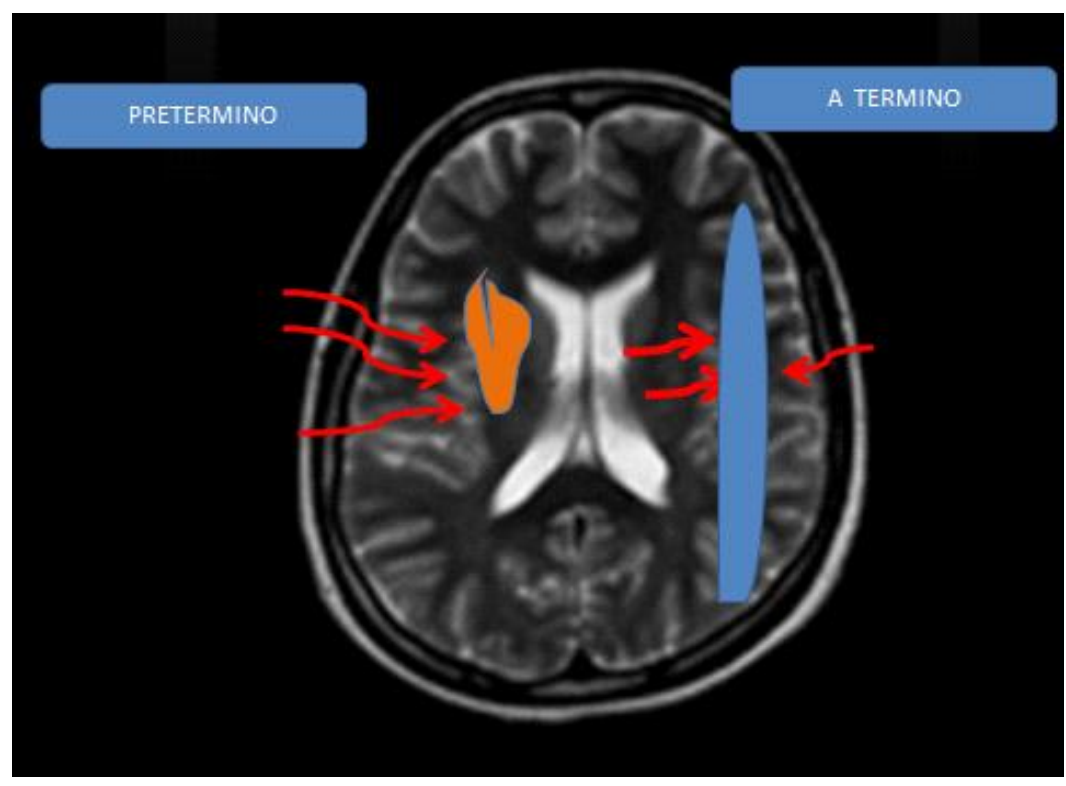

Fuente: Grupo de investigación. 
Factores extravasculares: Incluyen el tono vascular deficiente y el aumento de la actividad fibrinolítica que ocurren en situaciones como deshidratación y sepsis con coagulación intravascular diseminada.

Apgar: es un método de evaluación del estado del recién nacido $(\mathrm{RN})$, no se utiliza para la determinación de mortalidad o la morbilidad neonatal, valora la frecuencia cardíaca, el tono muscular y movimiento, madurez pulmonar, color de piel y oxigenación y respuesta a los reflejos. Los RN con un Apgar de 7 a 10 no necesitan intervención, cuando el RN tiene un puntaje menor es necesaria una evaluación adicional y una posible intervención (McKee. 2018). En RN con Apgar menor a 6, se encontró cierta relación con hemorragia grado IV (Cervantes-Ruiz et al., 2012).

Factores de origen materno: Preeclampsia, hemorragia intraparto, restricciones del crecimiento (Haque et al., 2008), anomalías placentarias, distocias en el trabajo de parto, corioamnionitis (Sánchez \& Gómez, 2007; Gago-Cabrera, 2018), trastornos cardiológicos, paradas cardiorespiratorias, neumopatías crónicas.

Respecto al parto prematuro la mayoría de ellos son espontáneos, por rotura prematura de membranas, en pocas ocasiones por insuficiencia cervical y en un menor grado por otras patologías maternas o fetales. La Organización Mundial de la Salud (OMS) clasifica al parto prematuro en tres categorías: el pretérmino moderado a tardío va entre la semana 32 a 37, muy prematuro entre 28 a 32 y extremadamente prematuro menor a 28 semanas (OMS, 2018).

\section{Valoración de la anatomía cerebral}

La anatomía cerebral fetal es estudiada intraútero y al nacer, a través de varios métodos de imagen: entre los más utilizados están el ultrasonido y la resonancia magnética.

El método primario es el ultrasonido en el cual se confirma el desarrollo embrionario inicial adecuado a través de la visualización de la morfología cerebral y permite conocer los cambios cerebrales según la edad gestacional.

De inicio se realiza una exploración básica intraútero (Figuras 2-4) según las Guías ISUOG:

- Evaluación cualitativa: identifica estructuras en 3 planos axiales.

- Plano transventricular.

- Plano transtalámico.

- Plano transcerebelar. 
- Evaluación cuantitativa: se refiere a datos antropométricos diámetro biparietal, diámetro fronto-occipital, circunferencia cefálica, así como otros datos como la amplitud del atrium ventricular, diámetro cerebral transverso y cisterna magna.

Figura 2. Plano transtalámico, valora dos medidas básicas en la valoración del cráneo fetal el diámetro biparietal y circunferencia cefálica. Fuente: adaptado a partir de Callen, 2018.

\section{Biometría: cabeza fetal}
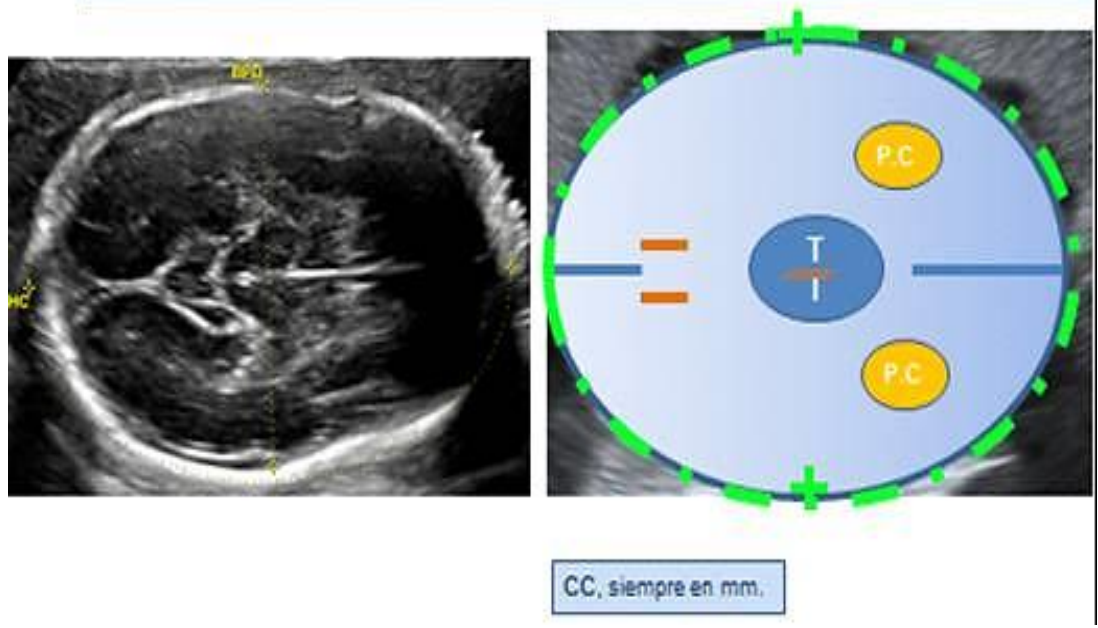

Fuente: Grupo de investigación.

Figura 3. Ultrasonido obstétrico, leve dilatación del ventrículo lateral.

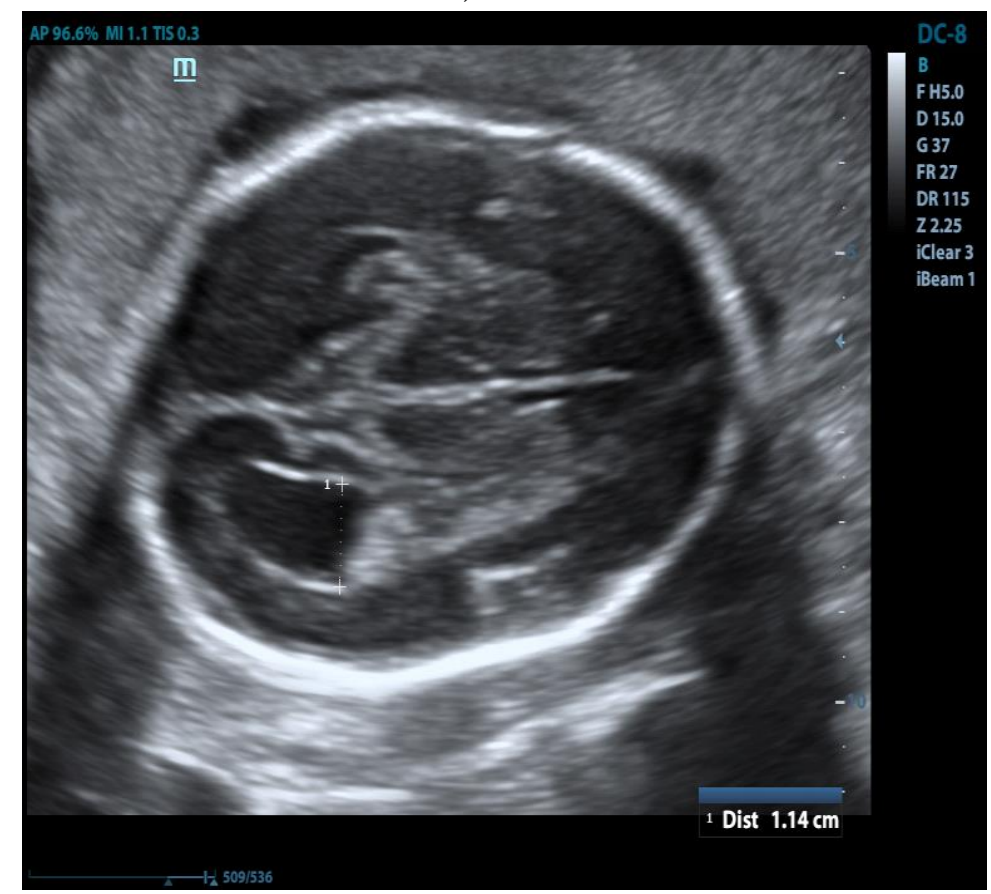

Fuente: Grupo de investigación. 
Figura 4. Ultrasonido obstétrico, se observa dilatación de ventrículos laterales y disminución del tamaño de los lóbulos frontales.

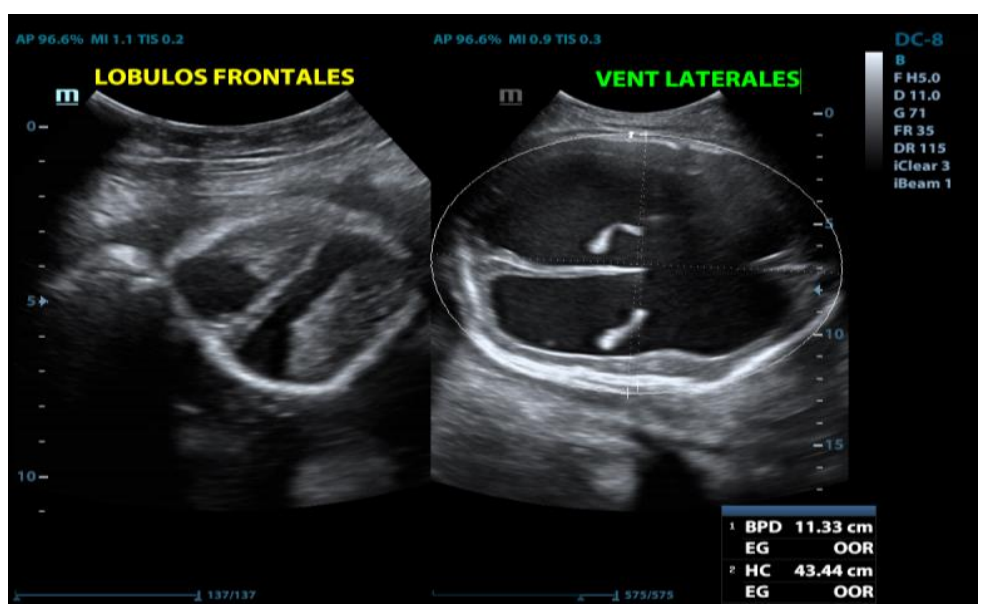

Fuente: Grupo de investigación.

\section{Técnica ultrasonográfica}

Asistidos por el personal de salud el estudio puede realizarse en la cuna, cumpliendo con estrictas normas de asepsia y con materiales estériles (Bhat \& Bhat, 2014; Llorens \& Moreno, 2018).

Los transductores sugeridos para efectuar la exploración son convexos, pequeños, con frecuencias entre 7 y $9 \mathrm{MHz}$, por adaptarse mejor a la fontanela. Transductores lineales de alta frecuencia $(10-18 \mathrm{MHz})$ valoran mejor el espacio extra parenquimatoso y del patrón de sulcación. La fontanela anterior es la ventana acústica más empleada por su amplitud y accesibilidad (Figura 5).

Figura 5. Tomografía de cráneo exhibe la fontanela anterior y la variación de tamaño. A. Neonato de 10 días. B. Neonato de 14 días. C. Lactante de cuatro meses de vida.
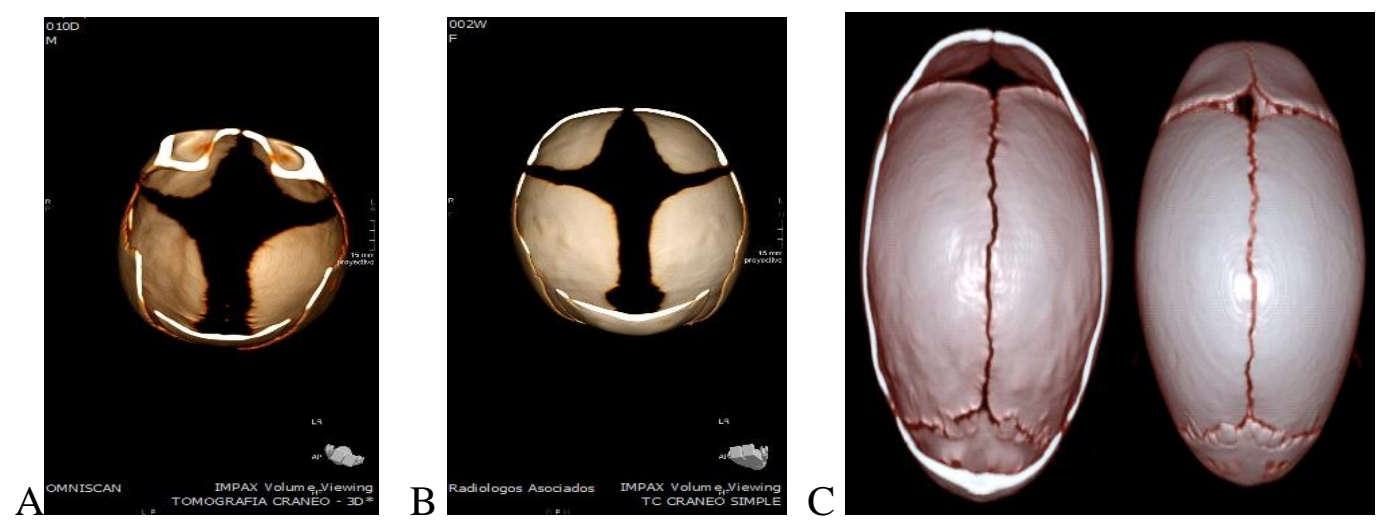

Fuente: Grupo de investigación. 
La fontanela posterior ofrece visualización de la fosa posterior, atrios de ventrículos laterales y la sustancia parietooccipital. La ventana mastoidea permite valorar con detalle la fosa posterior.

La ventana lateral o temporal valora las cisternas basales y el polígono de Willis (Llorens \& Moreno, 2016).

En los neonatos prematuros es mandatorio realizar estudio ultrasonográfico transfontanelar para determinar la existencia de hemorragia de la matriz germinal, ya que es un método extendido en el mundo y se puede realizar en cualquier momento y en la cama del paciente.

\section{Grados de hemorragia de la matriz germinal detectados por ultrasonido}

Observando la extensión de la hemorragia en el cerebro, se estableció un sistema en 1978 a través de los hallazgos de imagen por tomografía computarizada, que en la actualidad es el más usado y aceptado: la escala de Papile (Papile et al., 1978; Ayala et al., 2005).

A través del ultrasonido transfontanelar, Volpe (2008) introduce un sistema actualizado a partir de la clasificación Papile, en el que se especifican los hallazgos que mediante el ultrasonido deben reconocerse:

- Grado I o hemorragia de la matriz germinal con baja o mínima hemorragia intraventricular que corresponde a menos del $10 \%$ del área ventricular en proyección parasagital (Figuras 6-8). En ultrasonido se identifica una masa hiperecogénica por la presencia de coágulos con afectación de la hendidura caudalotalámica, el plexo coroideo luce engrosado en la región del trígono, esta imagen es observada por semanas a meses y paulatinamente se hace menos ecógenico, hasta que eventualmente se torna isoecogénica de dos a tres semanas, en ocasiones puede aparecer un quiste subependimario como secuela.

Figura 6. La hendidura caudo talámica, núcleo caudado (azul), Tálamo (amarillo), plexo coroideo (blanco).
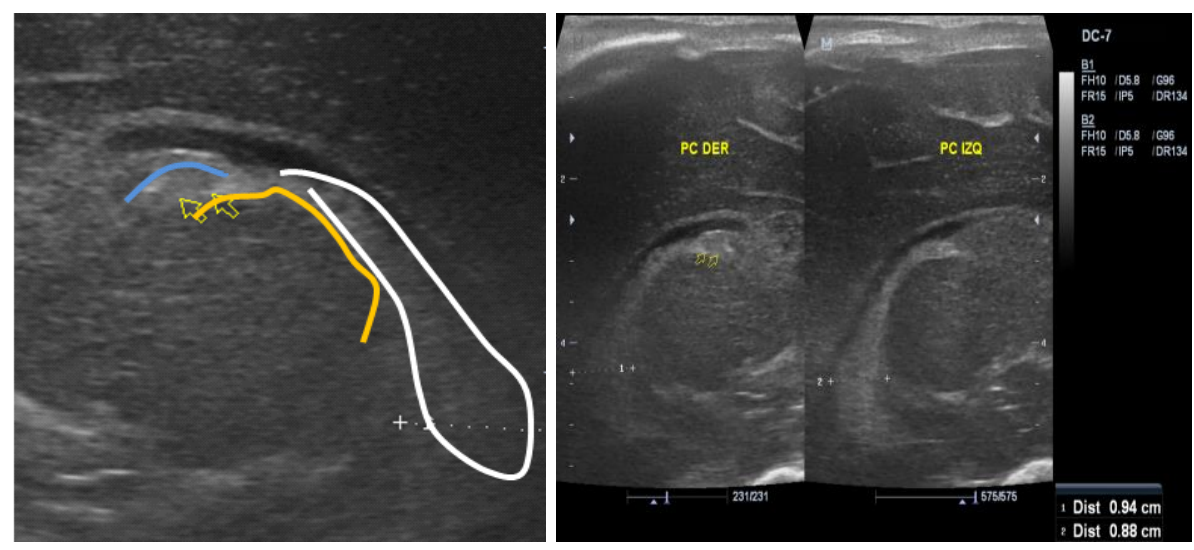

Fuente: Grupo de investigación. 
Figura 7. Vista coronal a través de la fontanela anterior, evidencia hemorragia de la matriz germinal, en el lado derecho se observa un quiste subpendimario.

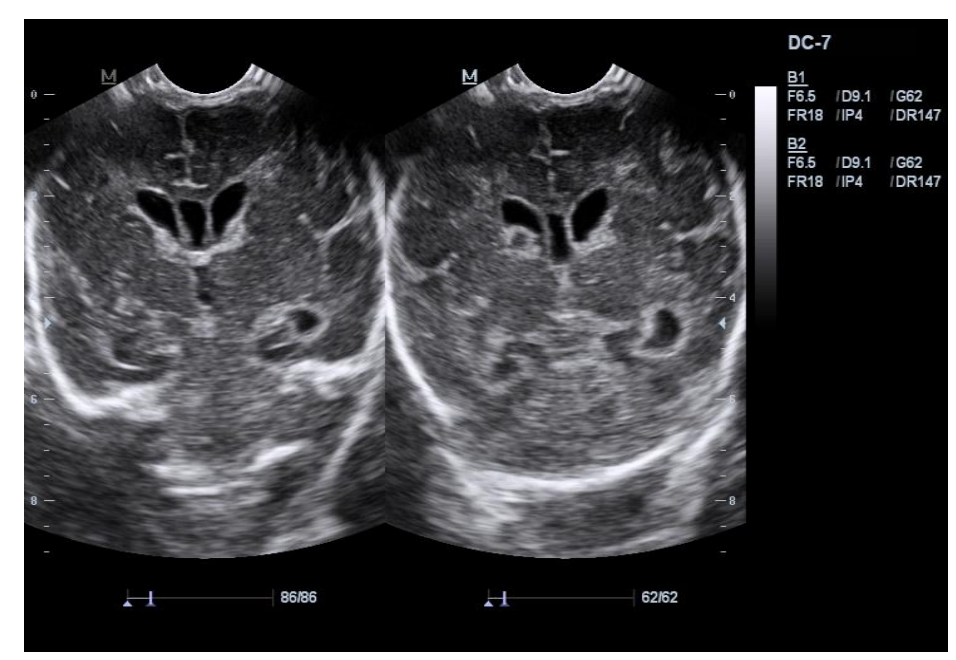

Fuente: Grupo de investigación.

Figura 8. Hemorragia de la matriz germinal, se demuestra hiperecogenicidad en los surcos caudotalámicos.

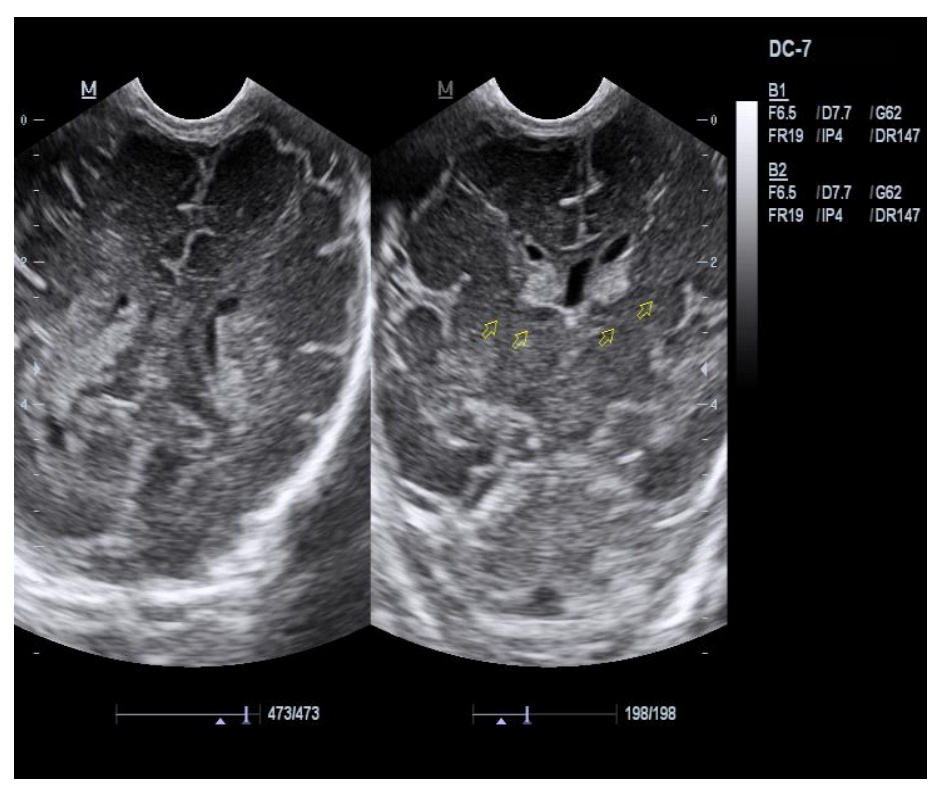

Fuente: Grupo de investigación.

- Grado II o hemorragia Intraventricular con un 10\% a 50\% del área ventricular lateral en proyección parasagital (Figura 9). La valoración a través de la fontanela posterior o axiales ayudan a caracterizar los hallazgos, si la hemorragia se extiende hacia la cisterna magna se incrementa el riesgo de una hidrocefalia posthemorrágica. 
Figura 9. Hemorragia intraventricular grado II, los restos hemáticos ocupan los ventrículos laterales en proporción menor al 50\%, no exhiben dilatación. Fuente: presente investigación.

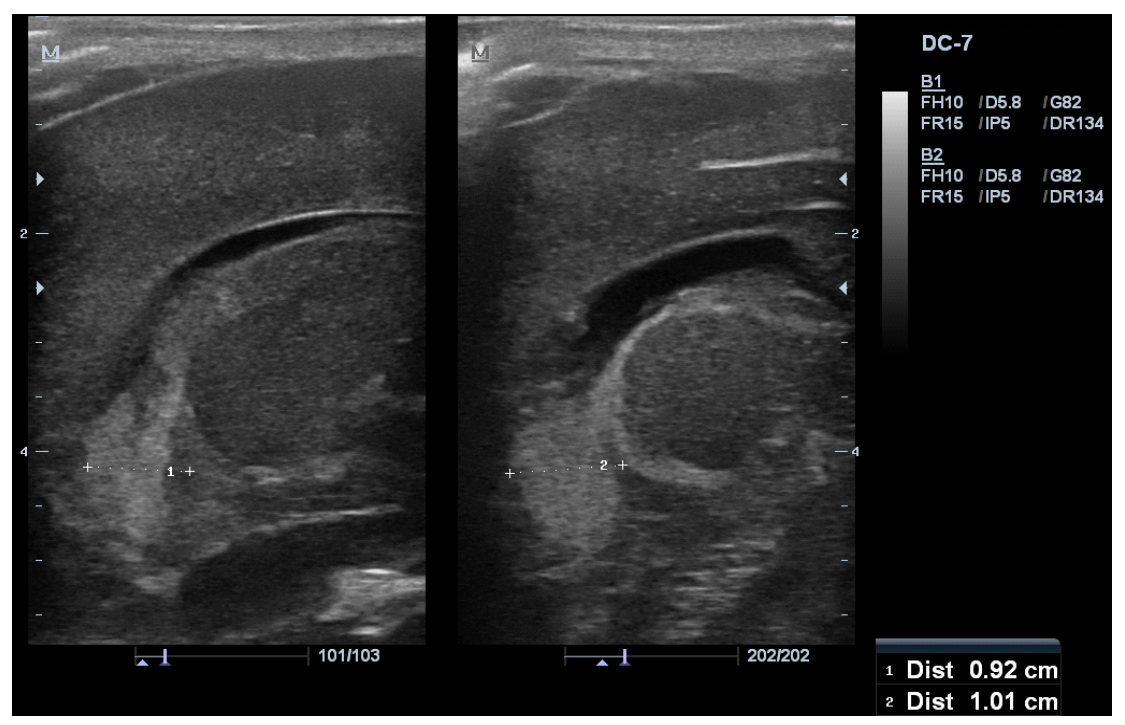

Fuente: Grupo de investigación.

- Grado III o hemorragia Intraventricular con ocupación mayor al 50\% del área ventricular en la misma proyección y usualmente distiende al ventrículo lateral, manifestándose con hidrocefalia (Figuras 10-12), con el transcurso del tiempo el coágulo se volverá más anecoico, la presencia de sangre en el líquido céfalo raquídeo (LCR) puede generar una ventriculitis química que se expresa con engrosamiento del recubrimiento subependimario.

Figura 10. Hemorragia asimétrica de los ventrículos laterales, se aprecia coágulo hiperecogénico que ocupa gran parte del ventrículo izquierdo.

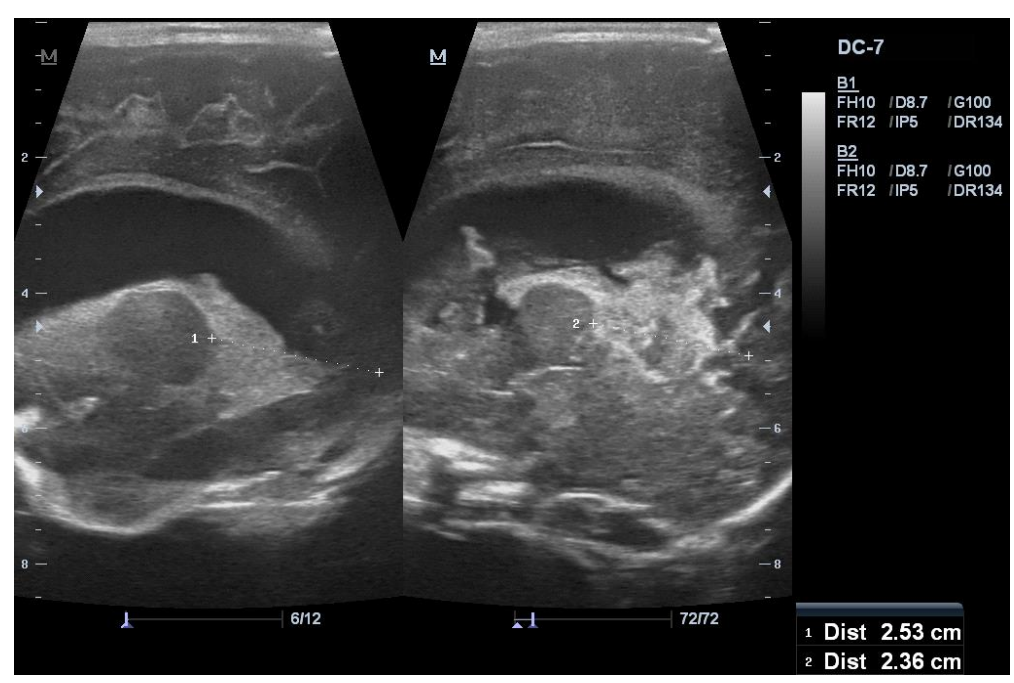

Fuente: Grupo de investigación. 
Figura 11. Dilatación de los ventrículos laterales y tercer ventrículo secundario a hemorragia intraventricular Grado III.

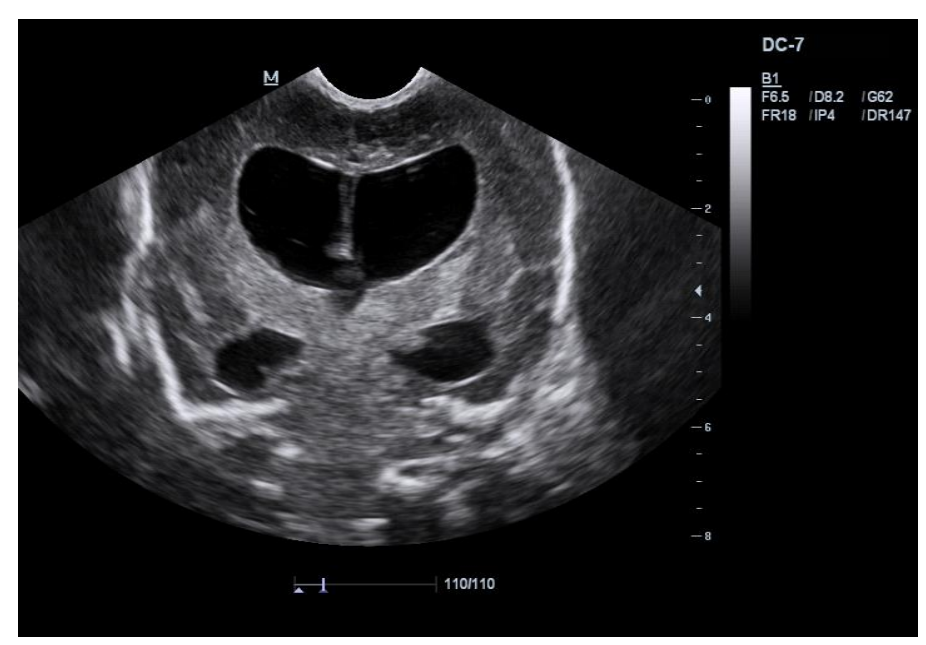

Fuente: Grupo de investigación.

Figura 12. Imagen parasagital del ventrículo lateral, es evidente el engrosamiento e hiperecogenicidad de la pared ventricular en relación con ventriculitis.

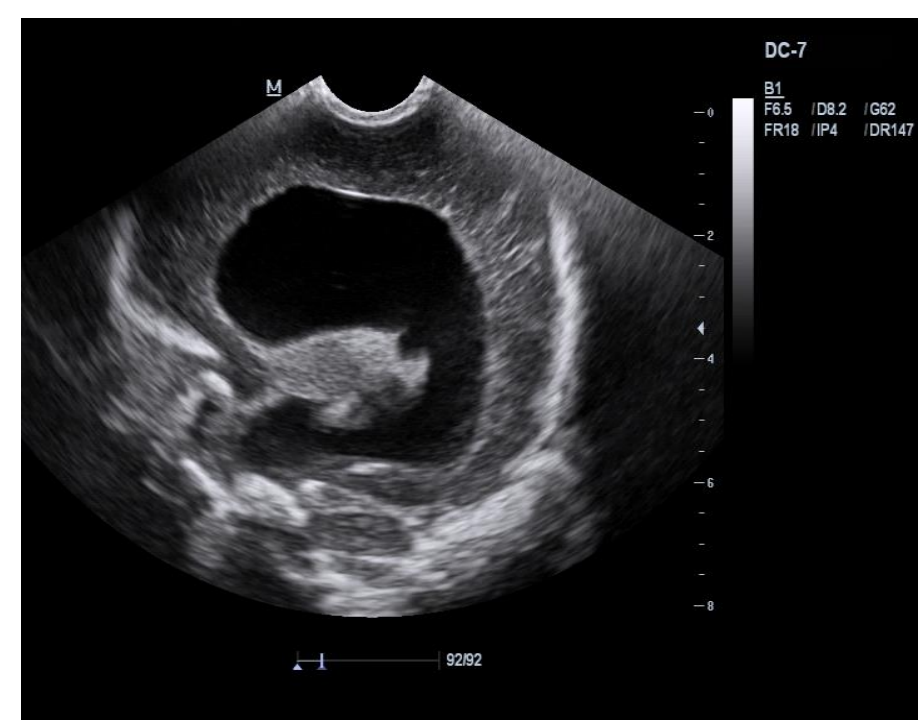

Fuente: Grupo de investigación.

- Grado IV, alteración parenquimatosa manifestada con hiperecogenicidad paraventricular (reportada, entre otros por Cinquegrani, 2014; Volpe, 2008). Se afectan con mayor frecuencia los lóbulos frontal y parietal (Figuras 13-15), probablemente por un infarto venoso hemorrágico o secundario a compresión de las venas subependimarias que origina infartos focales en la sustancia blanca periventricular de manera asimétrica y mayoritariamente unilaterales. (Rumack, 2014). 
Figura 13. Hemorragia parenquimatosa del lóbulo temporal y parietal derecha. Fuente: presente investigación.

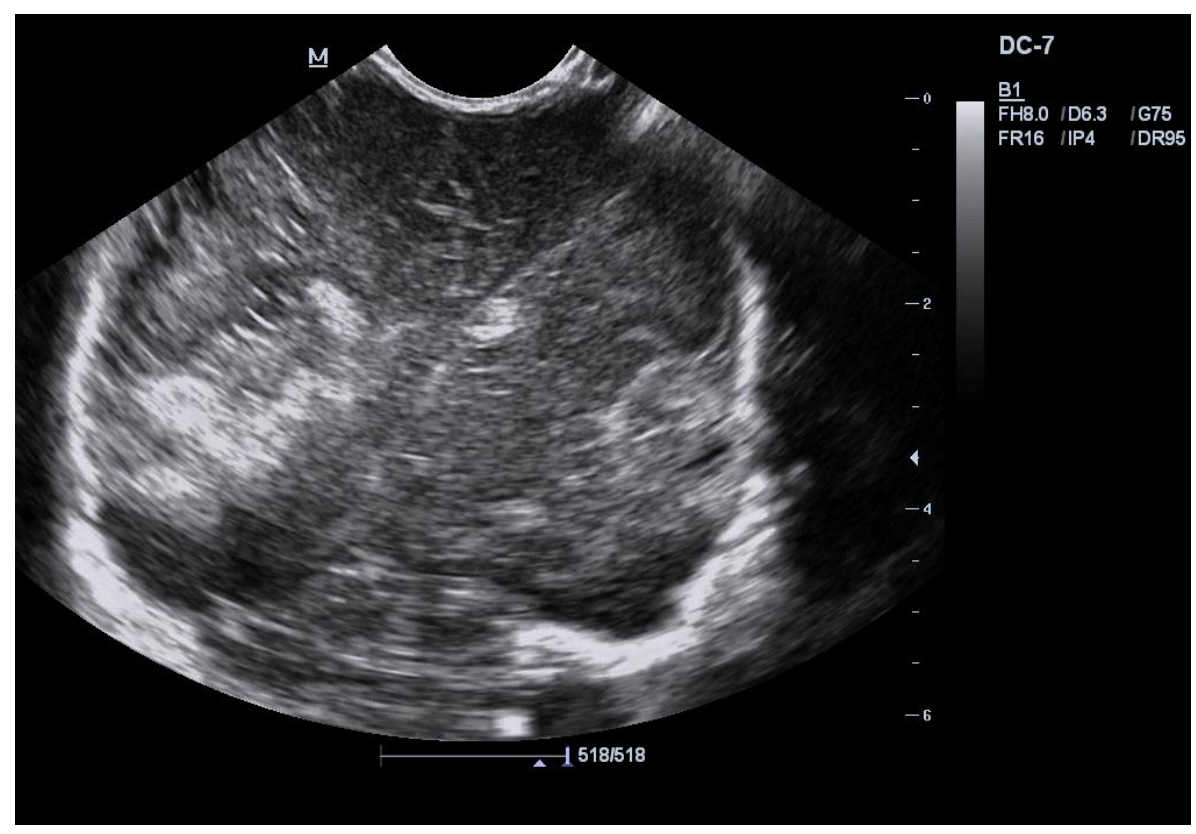

Fuente: Grupo de investigación.

Figura 14. Porencefalia secundaria a hemorragia parenquimatosa.

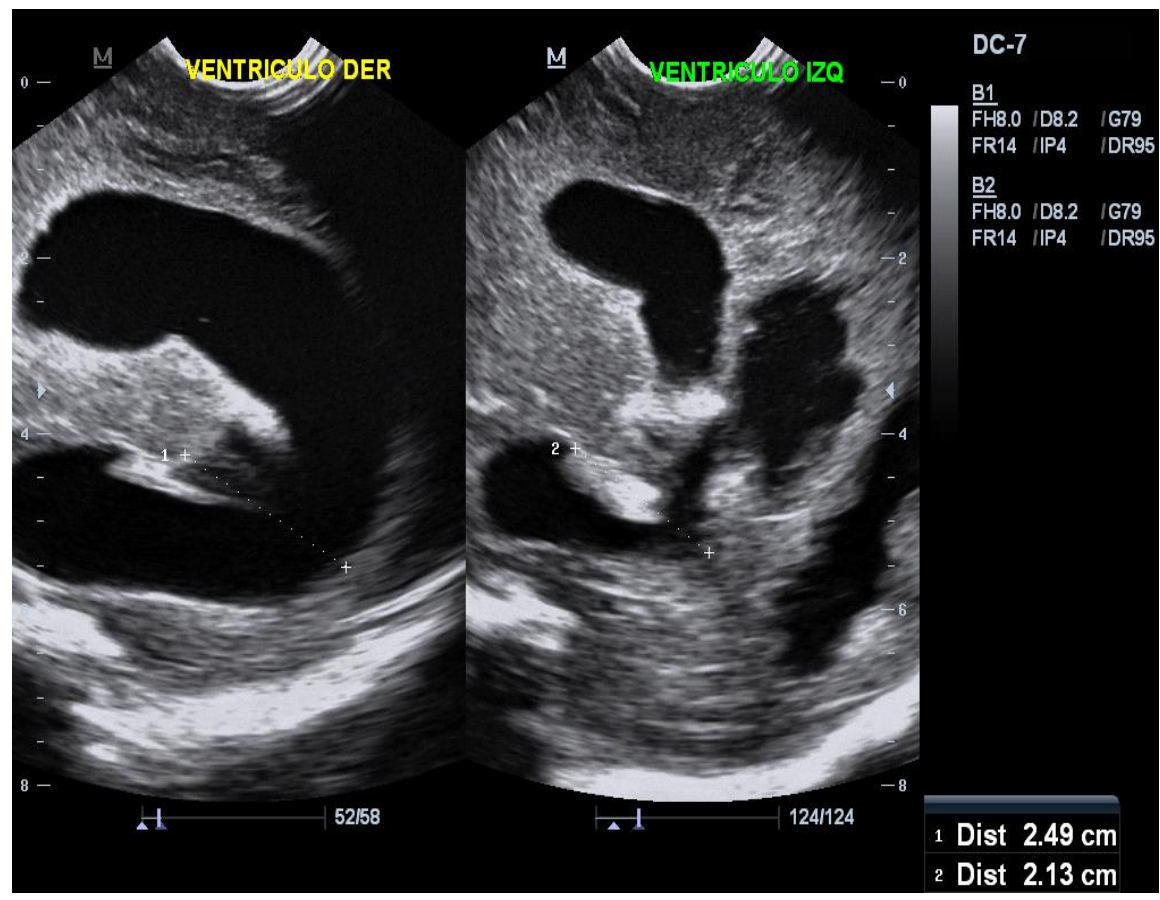

Fuente: Grupo de investigación. 
Figura 15. Seno venoso sagital con ausencia de flujo relacionado con trombosis, los espacios subaracnoideos son amplios. Fuente: presente investigación.

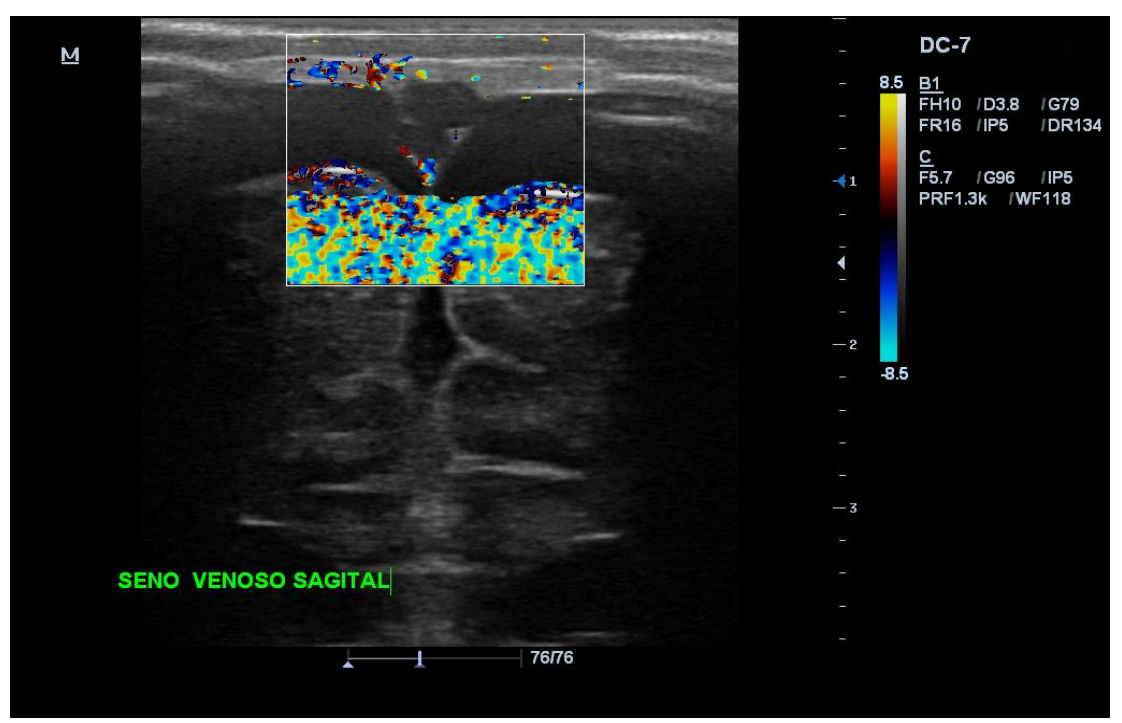

Fuente: Grupo de investigación.

\section{Leucomalacia Periventricular}

El ultrasonido transfontanelar tiene la capacidad de detectar algunas lesiones cerebrales en el recién nacido prematuro y diagnosticarlas con una sensibilidad importante, dentro de las cuales tenemos la hemorragia de la matriz germinal y leucomalacia periventricular (Chao et al., 2006).

La causa principal de la leucomalacia periventricular es precisamente eventos hipóxico - isquémicos; se conoce que en el recién nacido pretérmino el flujo Doppler cerebral tiene un patrón centrípeto, lo cual explica que las lesiones hipóxico isquémicas se presenten predominantemente en la sustancia blanca periventricular.

Para su diagnóstico es de gran utilidad tanto el ultrasonido como la resonancia magnética (Campistol et al.,1996).

Dentro de los hallazgos ecográficos están áreas hiperecogénicas que indican lesiones tempranas.

Los hallazgos ecográficos relacionados con la leucomalacia periventricular son:

a. De 1 a 2 semanas de vida: áreas hiperecogénicas de forma triangular periventricular.

$b$. De edad mayor o igual a la tercera semana de vida: se observan áreas anecoides. 
c. De edad mayor o igual al cuarto o quinto mes: dilatación ventricular. (Campistol et al.,1996).

\section{Conclusiones}

- La hemorragia de la matriz germinal es la primera causa de mortalidad en los recién nacidos prematuros en Ecuador, por lo que un diagnóstico oportuno es necesario para evitar las afectaciones en el neurodesarrollo del niño/a.

- El gold estándar para el diagnóstico de la hemorragia es el eco transfontanelar, el cual al ser operador - dependiente necesita de un radiólogo experto para la interpretación e identificación de los hallazgos en el mismo.

- El pronóstico correcto se basará en el grado de la hemorragia que se presente sabiendo que el Grado I ocupa menos del 10\% del área ventricular, mientras que el grado IV ya se acompaña con hidrocefalia.

- La utilización de diferentes herramientas imagenológicas, principalmente el eco transfontanelar, es un factor clave en el adecuado diagnóstico.

\section{Recomendaciones}

- Se recomienda el uso de esta técnica para el diagnóstico oportuno de la hemorragia de la matriz germinal y el grado de ésta en los recién nacidos que presenten síntomas o factores de riesgo.

- El eco transfantonelar siendo el gold estándar tiene limitaciones, una de ellas es que requiere de un experto para la identificación e interpretación de los hallazgos; por lo que resulta ineludible la interpretación de los resultados imagenológicos por personal capacitado.

\section{Referencias bibliográficas}

Ayala Mendoza, A. M., Carvajal Kalil, L. F., Carrizosa Moog, J., Galindo Hernández, A., \& Cornejo Ochoa, J. W. (2005). Hemorragia intraventricular en el neonato prematuro. Iatreia, 18(1), 71-77.

Ballabh, P. (2010). Intraventricular Hemorrhage in Premature Infants: Mechanism of Disease. Pediatr Res 67, 1-8. DOI: https://doi.org/10.1203/PDR.0b013e3181c1b176

Bhat, V., \& Bhat, V. (2014). Neonatal neurosonography: A pictorial essay. The Indian Journal of Radiology \& Imaging, 24(4), 389.

Cabañas F, \& Pellicer A. Protocolos de la asociación española de pediatría: protocolos de Neonatología. Lesión cerebral en el niño prematuro. Disponible en: http://www.aeped.es/sites/default/files/documentos/27.pdf 
Callen, P. (2018). Ecografía en obstetricia y ginecología. Buenos Aires: Médica Panamericana.

Campistol, J., Escofet, P., \& Póo, P. (1996). Leucomalacia periventricular: Diagnóstico retrospectivo en niños con diplejía espástica. Anales Españoles de Pediatría, 44, 553-556.

Cervantes-Ruiz, M. A., Rivera-Rueda, M. A., Yescas-Buendía, G., Villegas-Silva, R., \& Hernández-Peláez, G. (2012). Hemorragia intraventricular en recién nacidos pretérmino en una Unidad de Tercer Nivel en la Ciudad de México. Perinatología y reproducción humana, 26(1), 17-24.

Chao, C., Zaleski, G., \& Patton, A. (2006). Neonatal Hypoxic-Ischemic Encephalopathy: Multimodality Imaging Findings. RadioGraphics, 26, 159-172.

Cinquegrani, M. (2014). Hallazgo ecográfico de hemorragia intracraneal fetal en paciente con preeclampsia: Reporte de un caso. Congreso Argentino de Diagnóstico por Imágenes. Disponible en: http://congreso.faardit.org.ar/uploads/2014/poster/2014_499_PE_Miscelaneas.p df

Ertan, A. K., Tanriverdi, H. A., Meier, M., \& Schmidt, W. (2006). Perinatal risk factors for neonatal intracerebral hemorrhage in preterm infants. European Journal of Obstetrics \& Gynecology and Reproductive Biology, 127(1), 29-34.

Gago, I. (2018). Factores perinatales asociados a mortalidad en recién nacidos con peso menor de 1500 gramos hospitalizados en la unidad de cuidados intensivos neonatal del Hospital Nacional Sergio E. Bernales en el periodo de enero 2016 a junio del 2017.Universidad Ricardo Palma, Lima.

Haque, K. N., Hayes, A. M., Ahmed, Z., Wilde, R., \& Fong, C. Y. (2008). Caesarean or vaginal delivery for preterm very-low-birth weight $(\leq 1,250 \mathrm{~g})$ infant: experience from a district general hospital in UK. Archives of gynecology and obstetrics, 277(3), 207-212.

Jara-Santamaría, J., Flores, N., Tufiño, P., \& Yánez-Moretta, P. (2018). Estudio CURIE2017: Nociones de los estudiantes del internado rotativo de medicina del Hospital Carlos Andrade Marín (Quito) con respecto al uso y aplicación de estudios imagenológicos. Revista de la Federación Ecuatoriana de Radiología e Imagen, 11(2), 31-35.

Llorens, R., \& Moreno, A. (2016). El ABC de la ecografía transfontanelar y más. Radiología, 58, 129-141.

McKee-Garrett, T. (2013). Overview of the routine management of the healthy newborn infant. Up To Date.

MSP - Ministerio de Salud Pública del Ecuador. (2015). Guía de práctica clínica del RN prematuro. Disponible en: https://www.salud.gob.ec/wpcontent/uploads/2014/05/GPC-Rec\%C3\%A9n-nacido-prematuro.pdf

Montalvo-Ramos, P., Jara-Santamaría, J., Flores-Santamaría, N., Cornejo-Castro, P., \& Yánez-Moretta, P. (2018). Tratamiento de fracturas vertebrales por compresión con implante intramedular expandible SpineJack®: primera experiencia en Ecuador. Revista Ecuatoriana de Medicina y Ciencias Biológicas, 39(2), 129-133. 
Montejo, I., Moreno, C., Marin, A., Cárdenas, M., Artasona, L., \& Gambau, J. (2014). Alteraciones en el desarrollo de la corteza cerebral. Presentación clínica y hallazgos en TC y RM. Seram, S-0869.

Moore, K., \& Persaud, M. (2009). Embriología clínica. Madrid: Elsevier Science.

OMS. (2018). Nacimientos prematuros. Disponible en: http://www.who.int/mediacentre/factsheets/fs363/es/

Papile, L. A., Burstein, J., Burstein, R., \& Koffler, H. (1978). Incidence and evolution of subependymal and intraventricular hemorrhage: a study of infants with birth weights less than 1,500 gm. The Journal of pediatrics, 92(4), 529-534.

Pazmiño-Tandazo, P., Heredia-Aguirre, S., \& Yánez-Moretta, P. (2019). Factores de riesgo relacionados con deficiencia nutricional en niños de una comunidad centro andina de Ecuador. Revista Científica "Conecta Libertad" ISSN 2661-6904, 3(2), 13-23.

Rumack, C. (2014). Diagnóstico por Ecografía. Mosby Inc.

Sánchez Andrés, A., \& Gómez Tébar, M. (2007). Morbilidad neonatal en el parto instrumentado: mención especial a la ventosa obstétrica. Acta Pediátrica Española, 65(7), 322-326.

Segovia Morales, O. L., Latorre Latorre, J. F., Rodríguez Hernández, J., \& Pérez Vera, L. A. (2003). Hemorragia intraventricular en niños pretérmino, incidencia y factores de riesgo. Un estudio de corte transversal. MedUNAB, 57-62.

Volpe, J. J. (2008). Neurology of the newborn E-book. Philadelphia: Elsevier Health Sciences. 


\section{PARA CITAR EL ARTÍCULO INDEXADO:}

Jara Santamaría, J. C., Torres Morán, L. E., Trujillo Jácome, C. C., Yánez Moretta, A. P., Cumbicus Naranjo, G. F., \& Mantilla Naranjo, S. G. (2020). Hemorragia de la matriz germinal: puede ser detectada de manera efectiva. Anatomía Digital, 3(3), 26-43. https://doi.org/10.33262/anatomiadigital.v3i3.1328

\section{\Ciencia}

El artículo que se publica es de exclusiva responsabilidad de los autores y no necesariamente reflejan el pensamiento de la Revista Anatomía Digital.

El artículo queda en propiedad de la revista y, por tanto, su publicación parcial y/o total en otro medio tiene que ser autorizada por el director de la Revista Anatomía Digital.
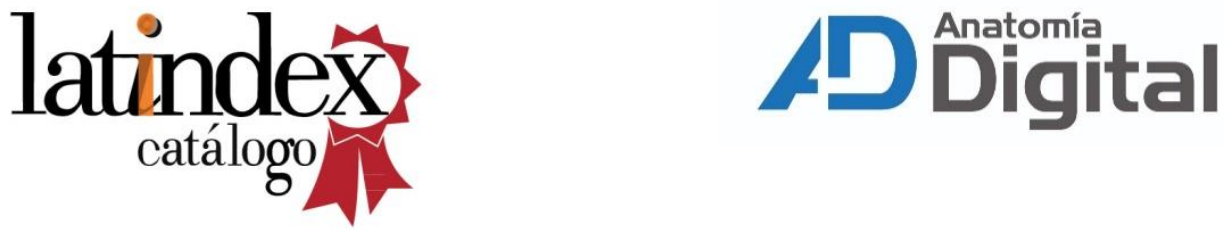